\title{
Fabrication of Hierarchical Sn-Beta Zeolite as Efficient Catalyst for Conversion of Cellulosic Sugar to Methyl Lactate
}

Bo Tang ${ }^{1}$, Shuang Li ${ }^{1}$, Wei-Chao Song ${ }^{1}$, En-Cui Yang ${ }^{1, *}$, Xiao-Jun Zhao ${ }^{1, *}$, Naijia Guan ${ }^{2}$, and Landong $\mathrm{Li}^{2}$,*

1 Key Laboratory of Inorganic-Organic Hybrid Functional Material Chemistry, Ministry of Education, Tianjin Key Laboratory of Structure and Performance for Functional Molecules, Tianjin Normal University, 393\# Binshui West Road, Xiqing District, Tianjin 300387, China

${ }^{2}$ School of Materials Science and Engineering \& National Institute for Advanced Materials, Nankai University, 38\# Tongyan Road, Jinnan District, Tianjin 300350, China

Pages: S1-S6

Figures: S1-S7

Tables: S1 


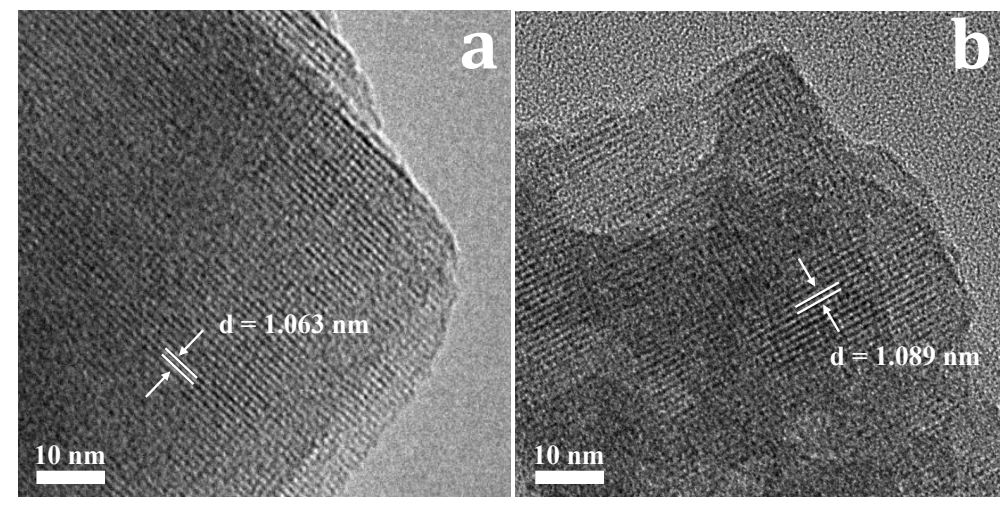

Figure S1 HR-TEM images of Si-Beta (a) and Sn-Beta-H4 (b) zeolites.

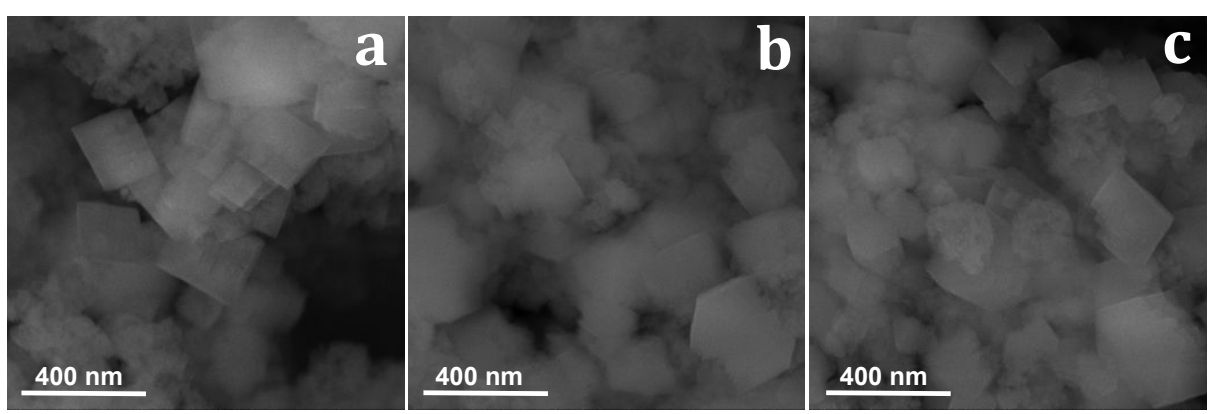

Figure S2 SEM images of Sn-Beta-H1 (a), Sn-Beta-H2 (b) and Sn-Beta-H4 (c) zeolites. 


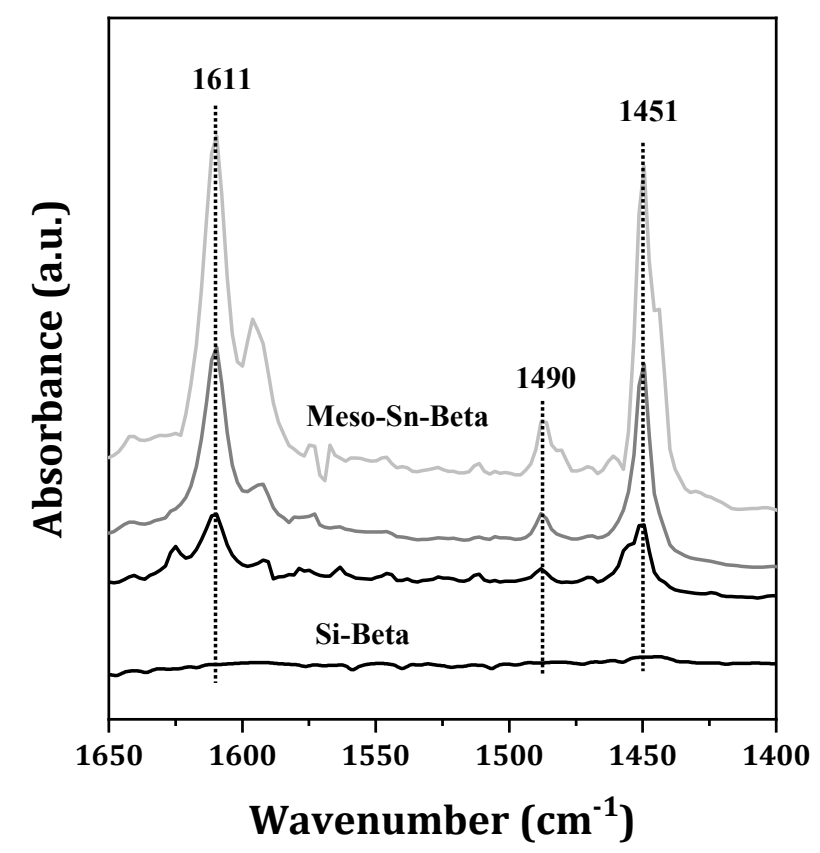

Figure S3 Pyridine-adsorption FTIR spectra of Si-Beta and Meso-Sn-Beta collected after evacuation treatment at 423 (light gray), 473 (gray) and $523 \mathrm{~K}$ (black).

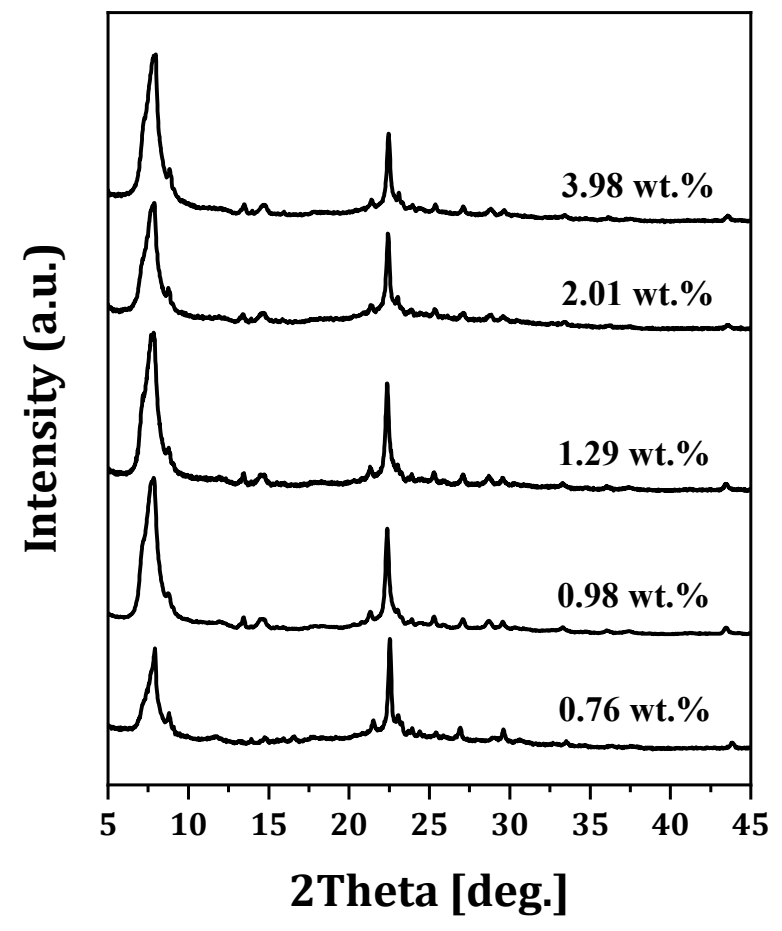

Figure S4 XRD patterns of Sn-Beta-H4 zeolites with different Sn loadings. 

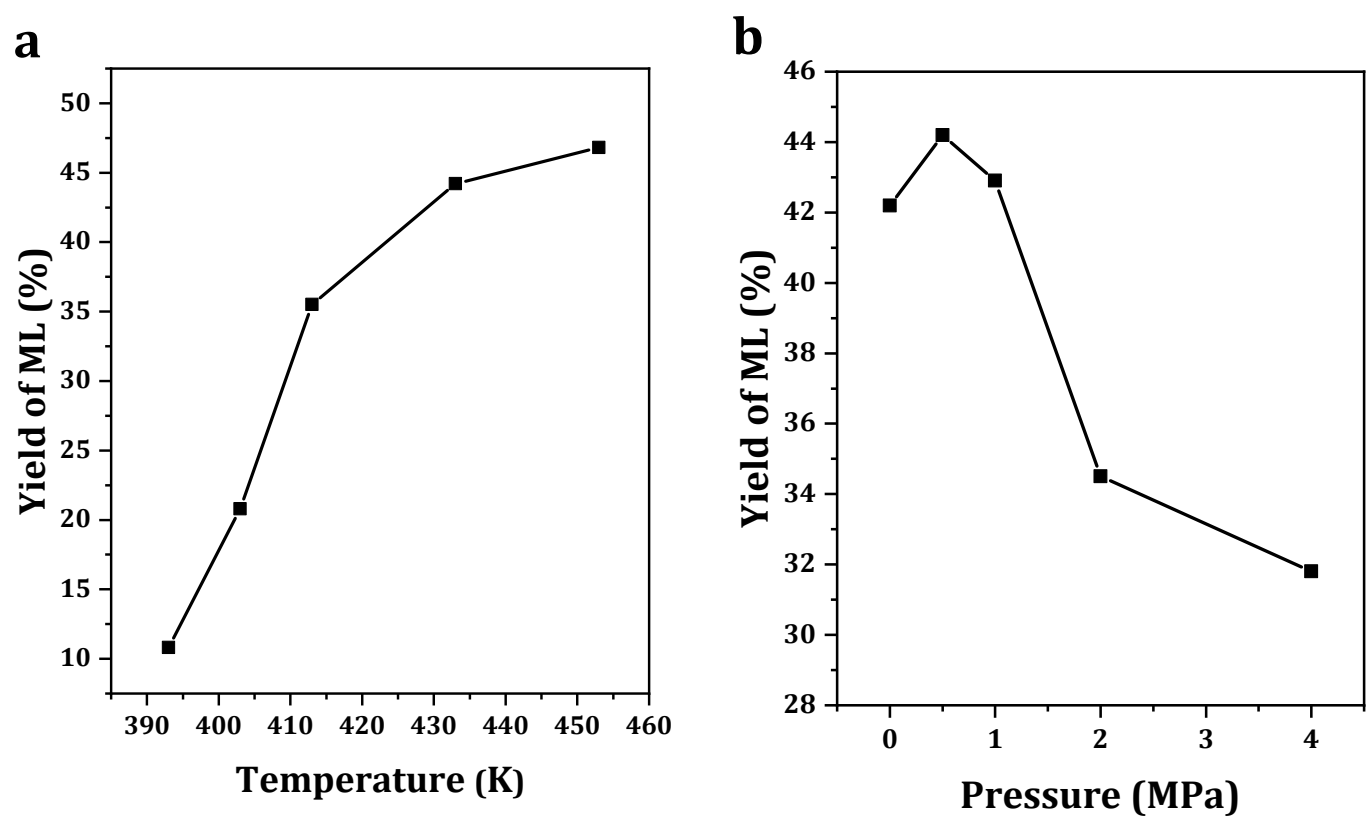

Figure S5 The impacts of reaction temperature (a) and pressure (b) on the catalytic conversion of glucose to methyl lactate over Sn-Beta-H4.

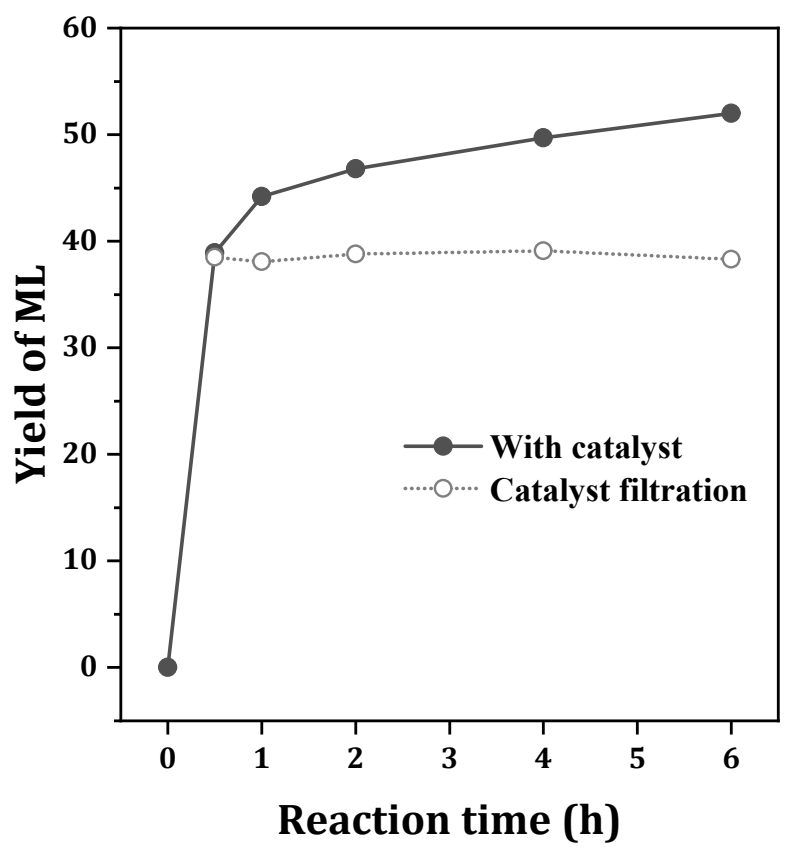

Figure S6 Time-dependent ML yield in the conversion of glucose over Sn-Beta-H4. Reaction conditions: glucose, $0.124 \mathrm{~g}$; methanol, $5 \mathrm{~mL}$; catalyst, $80 \mathrm{mg} ; \mathrm{N}_{2}$ pressure, $0.5 \mathrm{MPa}, 433 \mathrm{~K} ; 6 \mathrm{~h}$. 


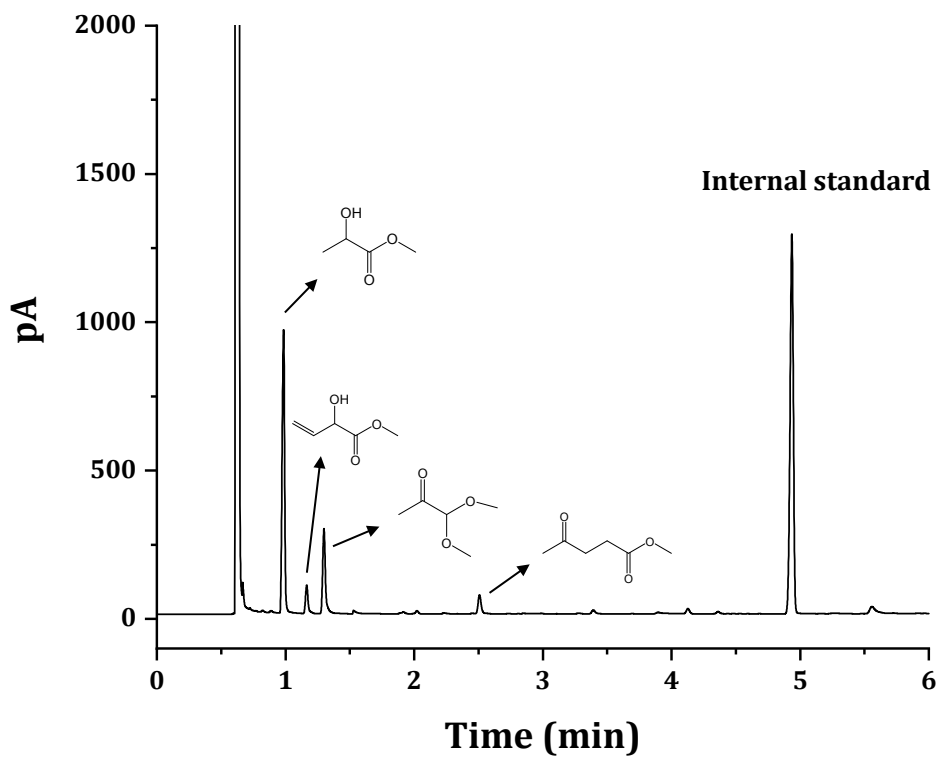

Figure S7 Typical chromatogram of glucose conversion catalyzed by Sn-Beta-H4.

Table S1 Physicochemical properties of Sn-Beta-H4 zeolite before and after use.

\begin{tabular}{llll}
\hline Sample & $n_{\mathrm{Si}} / n_{\mathrm{Sn}} \mathrm{ratio}^{a}$ & $\mathrm{SA}^{b}\left(\mathrm{~m}^{2} \mathrm{~g}^{-1}\right)$ & $\mathrm{PV}\left(\mathrm{cm}^{3} \mathrm{~g}^{-1}\right)$ \\
\hline Sn-Beta-H4 & 150 & 710 & 0.75 \\
Sn-Beta-H4 after 4 runs & 148 & 580 & 0.63 \\
Sn-Beta-H4 after calcination & 148 & 715 & 0.74 \\
\hline
\end{tabular}

${ }^{a}$ Determined by ICP. ${ }^{b}$ Obtained by the BET method. 